\title{
Rhodium Nanoparticle Shape Dependence in the Reduction of NO by CO
}

\author{
James Russell Renzas • Yawen Zhang • \\ Wenyu Huang • Gabor A. Somorjai
}

Received: 13 July 2009/Accepted: 26 August 2009/Published online: 11 September 2009

(C) The Author(s) 2009. This article is published with open access at Springerlink.com

\begin{abstract}
The shape dependence of the catalytic reduction of nitric oxide by carbon monoxide on rhodium nanopolyhedra and nanocubes was studied from 230 to $270{ }^{\circ} \mathrm{C}$. The nanocubes are found to exhibit higher turnover frequency and lower activation energy than the nanopolyhedra. These trends are compared to previous studies on $\mathrm{Rh}$ single crystals.
\end{abstract}

Keywords Catalysis - Nanoparticles - Nanocubes · $\mathrm{NO} \cdot \mathrm{CO} \cdot$ Rhodium $\cdot$ Shape dependence

\section{Introduction}

In recent years, intense efforts have been directed towards the development of a molecular understanding of the role of nanoparticle properties such as size, structure, and composition on catalytic activity and selectivity [1,2]. Nanoparticle shape, in particular, has been demonstrated to play an important role in catalysis [3-8]. The role of shape is thought to be closely related to the role of structure-sensitivity in catalysis. As an example, a study of benzene hydrogenation on $\sim 12-13 \mathrm{~nm}$ Pt nanoparticles found that both cyclohexene and cyclohexane were formed on cuboctahedral nanoparticles, which are dominated by

J. R. Renzas · W. Huang · G. A. Somorjai $(\bowtie)$

University of California and Lawrence Berkeley National

Laboratory, Berkeley, CA, USA

e-mail: somorjai@berkeley.edu

\section{J. R. Renzas}

e-mail: jrenzas@berkeley.edu

Y. Zhang

Peking University, Beijing, People's Republic of China 111-faces, whereas only cyclohexane was formed on 100dominated cubic nanoparticles [5]. These selectivities were consistent with previous results on (111) and (100) Pt single crystals $[9,10]$. Nanoparticle shape was also shown to play an important role in selectivity in pyrrole hydrogenation on Pt nanopolyhedra and nanocubes [4]. This study found that although $n$-butylamine was the dominant product for both 5 and $9 \mathrm{~nm} \mathrm{Pt}$ nanocubes regardless of temperature, both pyrrolidine and $n$-butylamine were formed on $5 \mathrm{~nm}$ Pt nanopolyhedra. These studies demonstrate the importance of the role of nanoparticle shape in catalysis and, in particular, the importance of characterizing this role in relation to single crystal studies on structure-sensitive reactions.

The reaction studied here, the catalytic reduction of nitric oxide (NO) by carbon monoxide (CO) on rhodium metal, is extremely important because of its role in the three-way catalytic converter used in automobile exhaust systems [11]. The reaction proceeds much faster on $\mathrm{Rh}$ than on Pd or Pt [12] and is known to be structure sensitive on $\mathrm{Rh}$ single crystals [13]. The products of the reaction are $\mathrm{CO}_{2}$ at all temperatures, $\mathrm{N}_{2} \mathrm{O}$ primarily $(\sim 75 \%)$ at the lower $\left(<320{ }^{\circ} \mathrm{C}\right)$ temperatures investigated in this study, and $\mathrm{N}_{2}$ primarily at higher temperatures $\left(>320{ }^{\circ} \mathrm{C}\right)$ [12]. Although many mechanisms have been proposed for this reaction [14-20], it remains an open area of investigation [21, 22] due to its enormous complexity. One relatively simple model, proposed by Permana et al. [23], is as follows, where $\mathrm{S}$ refers to an available surface site:

$\mathrm{CO}_{(\mathrm{g})}+\mathrm{S} \leftrightarrow \mathrm{CO}_{(\mathrm{ads})}$,

$\mathrm{NO}_{(\mathrm{g})}+\mathrm{S} \leftrightarrow \mathrm{NO}_{(\mathrm{ads})}$,

$\mathrm{NO}_{(\text {ads })}+\mathrm{S} \rightarrow \mathrm{N}_{(\mathrm{ads})}+\mathrm{O}_{(\mathrm{ads})}$ 


$$
\begin{aligned}
& \mathrm{CO}_{(\mathrm{ads})}+\mathrm{O}_{(\mathrm{ads})} \rightarrow \mathrm{CO}_{2(\mathrm{~g})}+2 \mathrm{~S}, \\
& \mathrm{NO}_{(\mathrm{ads})}+\mathrm{N}_{(\mathrm{ads})} \rightarrow \mathrm{N}_{2} \mathrm{O}_{(\mathrm{g})}+2 \mathrm{~S}, \\
& 2 \mathrm{~N}_{(\mathrm{ads})} \rightarrow \mathrm{N}_{2(\mathrm{~g})}+2 \mathrm{~S} .
\end{aligned}
$$

More detailed models, such as that proposed by Chuang and Tan [24], account for the role of Rh oxidation in the reaction, which is very important in strongly oxidizing conditions and for smaller, more easily oxidized catalysts. Other models, such as that proposed by Avalos et al., account for experimental evidence of $\mathrm{N}$-island formation [25] and concerns that Step (6), above, may be too slow to adequately account for $\mathrm{N}_{2}$ production during reaction. In light of this, these authors suggest that the decomposition of an intermediate surface species, $\mathrm{N}-\mathrm{NO}$, is responsible for $\mathrm{N}_{2}$ formation during the reaction. Much work has also been done to elucidate the role of nanoparticle size on this reaction [26-28]. Oh and Eickel, for example, found that increased $\mathrm{Rh}$ particle size leads to a significant increase in turnover frequency (TOF), a decrease in calculated activation energy, and no significant effect on selectivity [26]. The role of nanoparticle shape in the NO-CO reaction, however, has not yet been addressed. This paper reports results of a study on the role of nanoparticle shape in this reaction. $\mathrm{Rh}$ nanopolyhedra and nanocubes were synthesized, characterized, and studied in reaction conditions similar to those used in previous studies on Rh single crystals [14, 17, 23]. Our results show a clear shape-dependence for the NO-CO reaction at low temperatures $\left(230-270{ }^{\circ} \mathrm{C}\right)$ based on observed differences in TOF and activation energy on the $\mathrm{Rh}$ nanopolyhedra and $\mathrm{Rh}$ nanocube catalysts.

\section{Experimental Details}

$\mathrm{Rh}$ NPs of (111)-oriented nanopolyhedra [29] and (100)oriented nanocubes [30] were synthesized using two similar techniques, both of which have been published previously. In brief, to make the nanopolyhedra, $0.00625 \mathrm{mmol}\left[\mathrm{Rh}(\mathrm{Ac})_{2}\right]_{2}$ and $0.25 \mathrm{mmol}$ poly(vinylpyrrolidone) ( $\mathrm{Mw}=55,000$, in terms of the repeating unit) were added to $20 \mathrm{ml}$ ethylene glycol in a $50 \mathrm{~mL}$ threenecked flask at room temperature. The solution was then heated to $100{ }^{\circ} \mathrm{C}$ and degassed for 20 min under vacuum while stirring, resulting in a green solution. The flask was then heated to $185{ }^{\circ} \mathrm{C}$ at $10{ }^{\circ} \mathrm{C} \mathrm{min}{ }^{-1}$, and maintained at this temperature for $2 \mathrm{~h}$ under Ar. When the reaction was complete, an excess of acetone was added at room temperature and the resulting $\mathrm{Rh}$ nanopolyhedra were separated by centrifugation and washed twice by precipitation/ dispersion [29].

The procedure used to synthesize $\mathrm{Rh}$ nanocubes was similar to that used to synthesize $\mathrm{Rh}$ nanopolyhedra, except that $0.2 \mathrm{mmol} \mathrm{RhCl}_{3}, 1 \mathrm{mmol}$ tetramethylammonium bromide, and $4 \mathrm{mmol}$ poly(vinylpyrrolidone) $(\mathrm{Mw}=24,000$, in terms of the repeating unit) were added to $20 \mathrm{~mL}$ ethylene glycol in a $50 \mathrm{~mL}$ three-necked flask at room temperature, which was evacuated at $80{ }^{\circ} \mathrm{C}$ for $20 \mathrm{~min}$, and heated at $185{ }^{\circ} \mathrm{C}$ for $1.5 \mathrm{~h} \mathrm{[30].} \mathrm{Both} \mathrm{the} \mathrm{nanopolyhedra}$ and nanocube samples were subsequently deposited onto $1 \times 1 \mathrm{~cm}^{2}$ pieces of Si wafer using the Langmuir-Blodgett technique, which has been described previously [29].

The shape, size, and lattice structure of the Rh nanocrystals were analyzed using a Philips FEI Tecnai 12 Transmission electron microscope (TEM) and Philips CM200/FEG high-resolution TEM (HRTEM), operated at 100 and $200 \mathrm{kV}$, respectively. Analysis of at least 50 particles from each synthesis determined that the sizes of the two types of nanoparticle catalysts are comparable, with the nanopolyhedra measuring approximately $6.5 \pm 0.6 \mathrm{~nm}$ in diameter and the nanocubes measuring approximately $6.2 \pm 0.6 \mathrm{~nm}$ in diameter, as shown in Fig. 1. Powder X-ray diffraction (XRD) patterns were recorded on a Bruker D8 GADDS diffractometer using Co-K $\alpha$ radiation $(\lambda=1.79 \AA)$. Both TEM and XRD indicated that the nanopolyhedra have predominantly (111) surface sites, whereas the nanocubes have predominantly (100) surface sites. The presence of some (111) and (220)oriented sites in the XRD is likely due to a combination of defects in the nanoparticles and, especially for the (111) peak, the sensitivity of XRD to bulk crystallinity in addition to surface orientation. Sample coverage, uniformity, and aggregation were checked before and after reaction using a Zeiss Ultra 55 Field Emission Scanning Electron Microscope. No significant surface melting or particle aggregation was observed within the limits of detection of SEM for nanoparticles subjected to a reaction temperature of $270{ }^{\circ} \mathrm{C}$. Nanoparticles subjected to higher reaction temperatures typically showed evidence of melting and aggregation. Prior to reaction, particles were difficult to image, presumably due to the PVP capping layer, which may have been partially removed during reaction [31] (Fig. 2).

Reaction studies were performed in batch mode with 8 Torr NO, 8 Torr CO, and 744 Torr He in a 1-L ultrahigh vacuum chamber with a base pressure of $1.0 \times 10-$ 8 Torr. These reactant pressures were chosen in order to be consistent with conditions used for high-pressure single crystal studies [14, 17, 23]. Sample temperature was measured with a Type-K thermocouple mounted on a $8-\mathrm{mm}^{2}$ piece of blank Si wafer on the same ceramic button heater (Momentive) used to heat the sample. Products were measured with an HP Series II gas chromatograph equipped with a thermal conductivity detector and separated with two columns in parallel-a $1 \mathrm{~m} \times 1 / 8^{\prime \prime}$ 60/80 Argon sieve (Supelco) to separate $\mathrm{N}_{2}, \mathrm{NO}$, and $\mathrm{CO}$, and a 

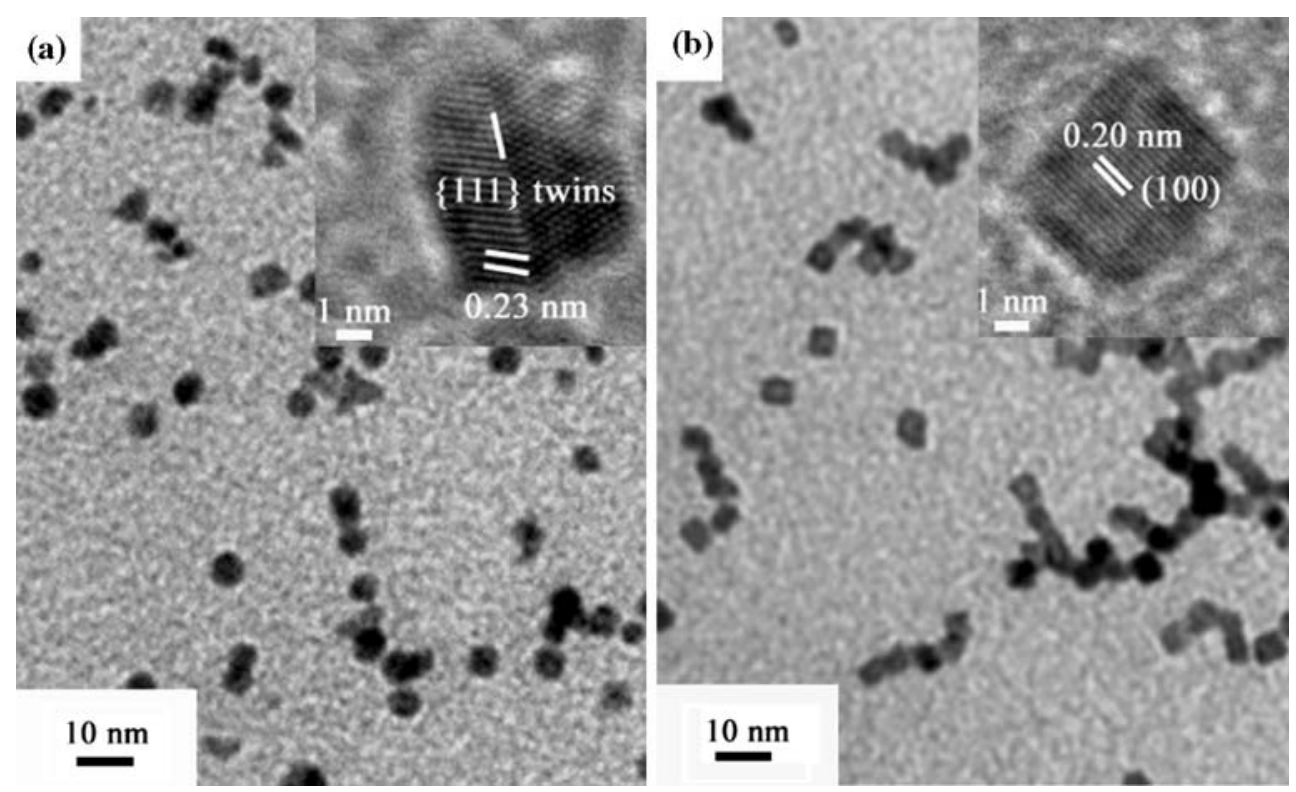

Fig. 1 TEM images of a $6.5 \mathrm{~nm}$ Rh nanopolyhedra, and b $6.2 \mathrm{~nm} \mathrm{Rh}$ nanocube catalysts

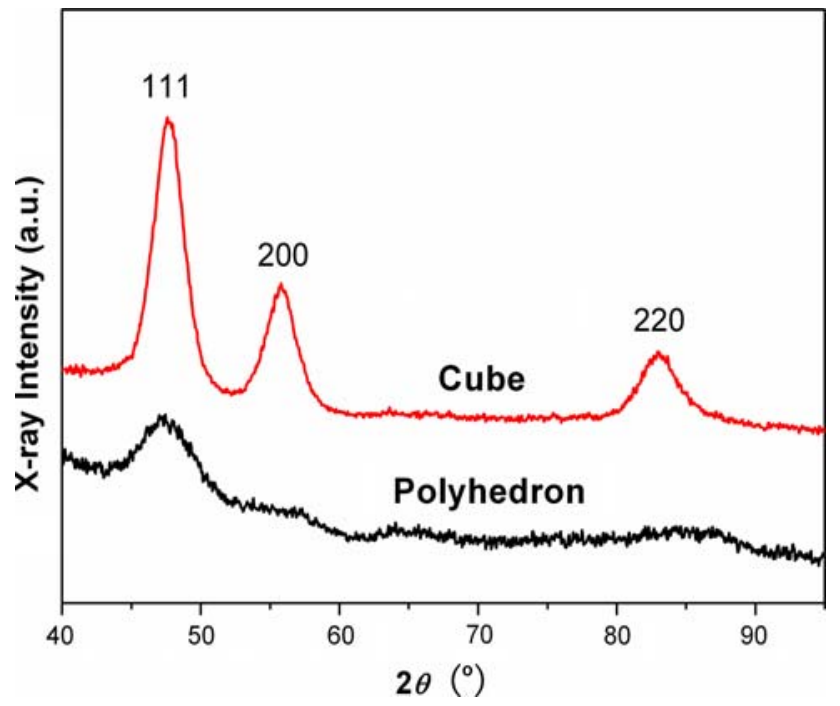

Fig. 2 XRD of Rh nanopolyhedra and nanocube catalysts

$3 \mathrm{~m} \times 1 / 8^{\prime \prime} 100 / 120$ Hayesep D (Supelco) to separate $\mathrm{N}_{2} \mathrm{O}$ and $\mathrm{CO}_{2}$. The reaction was carried out multiple times over fresh samples of each catalyst. The reaction temperature was kept between 230 and $270{ }^{\circ} \mathrm{C}$ to ensure that product formation was fast enough for accurate measurement and that the nanoparticles did not aggregate or sinter significantly. Data gathered above the $270{ }^{\circ} \mathrm{C}$ threshold was not reliable and is not reported here. TOF at each temperature was determined over the course of $3 \mathrm{~h}$. No significant deactivation was detected. The measured reaction rates are reported as TOFs and are measured in units of product molecules per metal surface site per second. The number of surface sites was determined from SEM images taken from randomly selected areas of the sample, analyzed with conventional image processing software to determine nanoparticle coverage, and converted into surface sites based purely on geometrical considerations. This method is intended to calculate the total number of surface sites and cannot account for site blocking by the PVP capping agent or other means. Consequently, the results presented here are overestimates of the number of active reaction sites and thus underestimate the true TOF. It is also possible that some PVP capping agent is removed during reaction [31], which could result in an increased number of active reaction sites at high temperature. Previous studies have established the difficulty of determining truly exact turnover numbers and the importance of qualitative analysis of TOFs even on comparatively stable samples in the same reaction system [32-34].

\section{Results and Discussion}

The TOF for $\mathrm{CO}_{2}, \mathrm{~N}_{2} \mathrm{O}$, and $\mathrm{N}_{2}$ formation for the $\mathrm{Rh}$ nanopolyhedra and $\mathrm{Rh}$ nanocube catalysts are reported in Fig. 3. The activation energies as calculated from the accumulation of each product from 230 to $270{ }^{\circ} \mathrm{C}$ are presented in Table 1 for both the nanopolyhedra and nanocube catalysts, as compared to single crystal data taken at similar temperatures and pressures $[13,17]$. The measured TOFs were higher for the nanocube catalysts than for the nanopolyhedra catalysts at all temperatures. The measured activation energies of the products from the reaction on the $\mathrm{Rh}$ nanopolyhedra catalysts were $37.0 \pm 3.3,39.3 \pm 4.3$, and $26.6 \pm 3.7 \mathrm{kcal} / \mathrm{mol}$ for $\mathrm{CO}_{2}, \mathrm{~N}_{2} \mathrm{O}$, and $\mathrm{N}_{2}$ formation, 

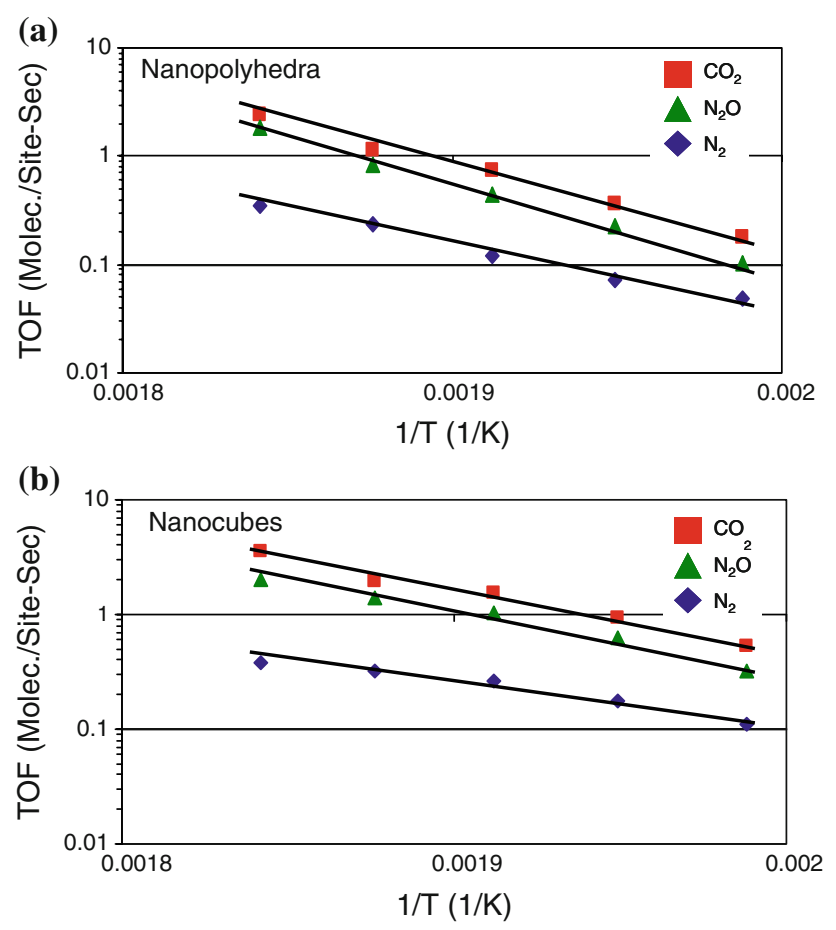

Fig. $3 \mathrm{CO}_{2}, \mathrm{~N}_{2} \mathrm{O}$, and $\mathrm{N}_{2}$ turnover frequencies of a Rh nanopolyhedra and $\mathbf{b} \mathrm{Rh}$ nanocube catalysts as a function of inverse temperature

Table 1 Apparent activation energies $\left(E_{\mathrm{a}}, \mathrm{kcal} / \mathrm{mol}\right)$ for $\mathrm{CO}_{2}, \mathrm{~N}_{2} \mathrm{O}$, and $\mathrm{N}_{2}$ and selectivity toward $\mathrm{N}_{2} \mathrm{O}$ (where Selectivity $=\mathrm{N}_{2} \mathrm{O}$ / $\left.\left(\mathrm{N}_{2} \mathrm{O}+\mathrm{N}_{2}\right)^{*} 100 \%\right)$ at $270{ }^{\circ} \mathrm{C}$ for the NO-CO reaction at 8 Torr NO and 8 Torr $\mathrm{CO}$ over $\mathrm{Rh}$ nanopolyhedra, $\mathrm{Rh}$ nanocube, $\mathrm{Rh}$ (111) single crystal, and Rh (100) single crystal catalysts

$$
\mathrm{CO}_{2} E_{\mathrm{a}} \quad \mathrm{N}_{2} \mathrm{O} E_{\mathrm{a}} \quad \mathrm{N}_{2} E_{\mathrm{a}} \quad \text { Selectivity }
$$

$\begin{array}{lllll}\text { Nanopolyhedra }^{\mathrm{a}} & 37.0 \pm 3.3 & 39.3 \pm 4.3 & 26.6 \pm 3.7 & 84 \\ \text { Nanocubes }^{\mathrm{a}} & 28.4 \pm 1.6 & 30.6 \pm 4.5 & 22.0 \pm 3.1 & 84 \\ \text { Rh (111) [17] } & 34.2 & 35.7 & 32.1 & \sim 72^{\mathrm{b}} \\ \text { Rh (100) LT } & 35.0 & 35.8 & 33.8 & \sim 64^{\mathrm{b}} \\ \quad \text { [13] } & & & & \\ \begin{array}{l}\text { Rh (100) HT } \\ \quad 22.2\end{array} & 15.3 & 26.2 & \\ \quad \text { [13] }\end{array}$

Single crystal values are as reported previously [13, 17]. LT refers to a temperature range of $\sim 255-320^{\circ} \mathrm{C}$ and $\mathrm{HT}$ refers to a range of $\sim 320-400{ }^{\circ} \mathrm{C}$

${ }^{\text {a }} E_{\mathrm{a}}(\mathrm{kcal} / \mathrm{mol})$ measured from 230 to $270{ }^{\circ} \mathrm{C}$

b Estimated from published results

respectively. The activation energies of the products from the reaction on the $\mathrm{Rh}$ nanocube catalysts were $28.4 \pm 1.6$, $30.6 \pm 4.5$, and $22.0 \pm 3.1 \mathrm{kcal} / \mathrm{mol}$, for $\mathrm{CO}_{2}, \mathrm{~N}_{2} \mathrm{O}$, and $\mathrm{N}_{2}$ formation. The selectivity for $\mathrm{N}_{2} \mathrm{O}$ versus $\mathrm{N}_{2}$ was similar for both catalysts and increased with temperature, from $\sim 70 \% \mathrm{~N}_{2} \mathrm{O}$ at $230{ }^{\circ} \mathrm{C}$ to $\sim 84 \% \mathrm{~N}_{2} \mathrm{O}$ at $270{ }^{\circ} \mathrm{C}$. Both catalysts produced primarily $\mathrm{N}_{2} \mathrm{O}$ in this temperature range.
The $\mathrm{Rh}$ nanopolyhedra and $\mathrm{Rh}$ nanocubes compare well to trends in $\mathrm{Rh}$ single crystals studied at similar pressures. Previous reports in this temperature range have found higher TOFs for Rh (100) single crystals than for Rh (111) single crystals $[13,17]$. Both $\mathrm{Rh}(100)$ and $\mathrm{Rh}$ (111) single crystals are strongly selective towards $\mathrm{N}_{2} \mathrm{O}$ in this temperature range $[13,17]$. The similarities in the trends between these single crystal results and the shape-dependent results presented here for (111)-dominated nanopolyhedra and (100)-dominated nanocubes indicate that the effect of surface structure on TOF and selectivity is similar in both nanoparticle and single crystal regimes. Evidence from measured activation energies, however, implies more complexity, as shown in Table 1. Although the activation energies for $\mathrm{CO}_{2}$ and $\mathrm{N}_{2} \mathrm{O}$ formation on the nanopolyhedra catalysts are similar to those previously reported for this reaction on $\mathrm{Rh}$ (111) single crystals [17], the measured activation energies for $\mathrm{CO}_{2}$ and $\mathrm{N}_{2} \mathrm{O}$ formation on the nanocube catalysts are intermediate to the two separate temperature regimes reported previously on $\mathrm{Rh}$ (100) single crystals [13]. Rh (100) single crystals have been reported to feature both a low temperature regime with higher activation energies for all products and a high temperature regime with lower activation energies for all products starting at about $315^{\circ} \mathrm{C}[13,15]$. Herman et al. attributed this breakpoint to a higher coverage of adsorbed $\mathrm{N}$ and adsorbed NO species on the (100) surface at lower temperatures and a consequent shortage of available sites for NO dissociation, a shortage which is alleviated at high temperatures to the extent that the rate of NO dissociation begins to limit the reaction rather than the availability of sites [13]. The activation energy measured for $\mathrm{N}_{2}$ formation on both the nanopolyhedra and nanocube catalysts was lower than that reported on single crystals [13], as reported in Table 1. This may be due to the increased selectivity towards $\mathrm{N}_{2} \mathrm{O}$ observed in the nanoparticle catalysts. In addition to the effect of $\mathrm{NO}$ adsorption and dissociation site availability, another possible explanation for the intermediate activation energies found for the nanocubes is that the small size of the nanoparticles leads to different kinetics than for (100) single crystals. In this case, the observed differences could be attributed to the role of $\mathrm{Rh}$ oxide in catalytic reactivity $[24,35,36]$, because relatively small transition metal nanoparticles develop stable oxides at lower temperatures than single crystals [37], which may amplify the effect of differences in the oxidation of $\mathrm{Rh}$ (111) and $\mathrm{Rh}$ (100) [38] on the catalytic reactivity of the $6.2 \mathrm{~nm}$ nanocubes and $6.5 \mathrm{~nm}$ nanopolyhedra.

Surface reconstruction and rearrangement is also a possible cause of the intermediate activation energies measured on the cubic nanoparticles, as single crystals are well-known to reconstruct at elevated temperatures and pressures [39] and nanoparticle surface melting is a 
growing part of present day inquiry [40]. Surface reconstruction is also known to be effected by surface oxidation in some cases [36], a phenomenon which may be important in the system investigated in this work. Although not presented in detail here, some experimental evidence during reaction above $270{ }^{\circ} \mathrm{C}$ and subsequent re-reaction at lower temperatures on the same $\mathrm{Rh}$ nanocube sample suggested surface reconstruction and a shift towards (111)-like kinetics in the nanocubes, which did not occur for the nanopolyhedra, which were already in the low surface energy (111) surface atom arrangement. In this case it is possible that the intermediate activation energies reported for the cubic nanocatalysts may have been a result of temperature-dependent surface reconstruction and the measurement of a temperature regime either intermediate to or inclusive of two separate crystal-face regimes-a (100)-dominated low temperature regime and a (111)dominated post-melting high temperature regime. Further work is required to fully understand the role of surface reconstruction in structure sensitive catalytic reactions on shape-controlled nanoparticles.

The differences in the overall catalytic activity between the nanopolyhedra and nanocubes are likely due to several mechanistic differences in the reaction between NO and CO on Rh (100) and Rh (111) [13-15, 17, 19, 21-23, 25, 41-46]. In summary, the reaction between CO and $\mathrm{O}$ is faster and the products of $\mathrm{NO}$ dissociation have a higher heat of adsorption on the (100) surface than on the (111) surface [41]. These differences mean that $\mathrm{CO}_{2}$ formation and NO dissociation are faster on (100) surfaces at low temperatures, which leads to higher TOFs for all products on (100) surfaces at relatively low temperatures. As temperature increases, however, the accumulation of strongly bound $\mathrm{N}$ leads to a lower rate of $\mathrm{N}_{2}$ formation and desorption, which eventually leads to site blocking and a greater degree of lateral interactions with coadsorbed species [46]. When the temperature is high enough $\left(\sim 320{ }^{\circ} \mathrm{C}\right.$ on the (100) single crystal), $\mathrm{N}_{2}$ is formed more easily and there is an abrupt change in selectivity towards $\mathrm{N}_{2}$, which also affects the formation of the other products [13].

\section{Conclusions}

The study of the role of shape is important to the understanding and development of new nanocatalysts for future applications. In this study, we showed a relationship between nanoparticle shape and catalytic properties for the reduction of $\mathrm{NO}$ by $\mathrm{CO}$. We synthesized PVP-capped $6.5 \mathrm{~nm}$ nanopolyhedra and $6.2 \mathrm{~nm}$ rhodium nanocubes and deposited them evenly across silicon wafers using the Langmuir-Blodgett technique. We then characterized the nanoparticles with TEM, XRD, and SEM, and studied their behavior in the $\mathrm{NO}+\mathrm{CO}$ reaction. Our study found that nanocubes show higher TOF and lower activation energies for all products and similar selectivity toward $\mathrm{N}_{2} \mathrm{O}$ between 230 and $270{ }^{\circ} \mathrm{C}$ as compared to nanopolyhedra due to the differences in surface structure. The activation energies for product formation on the $\mathrm{Rh}$ nanopolyhedra was found to be comparable to previous reports on $\mathrm{Rh}$ (111) single crystals, whereas the activation energies measured on the $\mathrm{Rh}$ nanocubes were found to be intermediate to those previously reported for two temperature regimes studied on $\mathrm{Rh}$ (100) single crystals. This study clearly shows that nanoparticle shape has an important effect on the reduction of NO by $\mathrm{CO}$ at low temperatures and implies that nanoparticle shape is an important criterion in the development of nanocatalysts for a wide range of structure-sensitive heterogeneous catalytic reactions.

Acknowledgements This work was supported by the Director, Office of Science, Office of Basic Energy Sciences, of the U.S. Department of Energy under Contract No. DE-AC02-05CH11231. Y.W.Z. gratefully acknowledges the financial aid of Huaxin Distinguished Scholar Award from Peking University Education Foundation of China.

Open Access This article is distributed under the terms of the Creative Commons Attribution Noncommercial License which permits any noncommercial use, distribution, and reproduction in any medium, provided the original author(s) and source are credited.

\section{References}

1. Bell AT (2003) Science $299: 1688$

2. Peng ZM, Yang H (2009) Nano Today 4:143

3. Lee I, Delbecq F, Morales R, Albiter MA, Zaera F (2009) Nat Mater 8:132

4. Tsung CK, Kuhn JN, Huang WY, Aliaga C, Hung LI, Somorjai GA, Yang PD (2009) J Am Chem Soc 131:5816

5. Bratlie KM, Lee H, Komvopoulos K, Yang PD, Somorjai GA (2007) Nano Lett 7:3097

6. Grass ME, Yue Y, Habas SE, Rioux RM, Teall CI, Yang P, Somorjai GA (2008) J Phys Chem C 112:4797

7. El-Sayed MA (2006) Abstr Pap Am Chem Soc 231:212

8. Subhramannia M, Pillai VK (2008) J Mater Chem 18:5858

9. Bratlie KM, Flores LD, Somorjai GA (2006) J Phys Chem B 110:10051

10. Bratlie KM, Kliewer CJ, Somorjai GA (2006) J Phys Chem B 110:17925

11. Taylor KC (1993) Catal Rev Sci Eng 35:457

12. Granger P, Dhainaut F, Pietrzik S, Malfoy P, Mamede AS, Leclercq L, Leclercq G (2006) Top Catal 39:65

13. Herman GS, Peden CHF, Schmieg SJ, Belton DN (1999) Catal Lett 62:131

14. Permana H, Ng KYS, Peden CHF, Schmieg SJ, Lambert DK, Belton DN (1996) J Catal 164:194

15. Hendershot RE, Hansen RS (1986) J Catal 98:150

16. Peden CHF, Goodman DW, Blair DS, Berlowitz PJ, Fisher GB, Oh SH (1988) J Phys Chem 92:1563 
17. Peden CHF, Belton DN, Schmieg SJ (1995) J Catal 155:204

18. Belton DN, DiMaggio CL, Schmieg SJ, Ng KYS (1995) J Catal 157:559

19. Avalos LA, Bustos V, Unac R, Zaera F, Zgrablich G (2005) J Mol Catal A Chem 228:89

20. Hecker WC, Bell AT (1983) J Catal 84:200

21. Matsushima T (2007) Phys Chem Chem Phys 9:3031

22. Gustafson J, Westerstrom R, Resta A, Mikkelsen A, Andersen JN, Balmes O, Torrelles X, Schmid M, Varga P, Hammer B, Kresse G, Baddeley CJ, Lundgren E (2009) Catal Today

23. Permana H, Ng KYS, Peden CHF, Schmieg SJ, Belton DN (1995) J Phys Chem 99:16344

24. Chuang SSC, Tan CD (1998) J Catal 173:95

25. Zaera F, Gopinath CS (2002) J Chem Phys 116:1128

26. Oh SH, Eickel CC (1991) J Catal 128:526

27. Araya P, Gracia F, Cortes J, Wolf EE (2002) Appl Catal B Environ 38:77

28. Kaspar J, Deleitenburg C, Fornasiero P, Trovarelli A, Graziani M (1994) J Catal 146:136

29. Zhang YW, Grass ME, Habas SE, Tao F, Zhang TF, Yang PD, Somorjai GA (2007) J Phys Chem C 111:12243

30. Zhang YW, Grass ME, Kuhn JN, Tao F, Habas SE, Huang WY, Yang PD, Somorjai GA (2008) J Am Chem Soc 130:5868

31. Grass ME, Zhang YW, Butcher DR, Park JY, Li YM, Bluhm H, Bratlie KM, Zhang TF, Somorjai GA (2008) Angew Chem Int Ed 47:8893
32. Choi KI, Vannice MA (1991) J Catal 131:1

33. Yao YFY (1984) J Catal 87:152

34. Yoon KJ, Kang HK, Yie JE (1997) Korean J Chem Eng 14:399

35. Gustafson J, Westerström R, Resta A, Mikkelsen A, Andersen JN, Balmes O, Torrelles X, Schmid M, Varga P, Hammer B, Kresse G, Baddeley CJ, Lundgren E, Catal Today (in press, corrected proof)

36. Medvedev VK, Suchorski Y, Voss C, de Bocarme TV, Bar T, Kruse N (1998) Langmuir 14:6151

37. Campbell CT (2006) Phys Rev Lett 96:066106/1

38. Seriani N, Mittendorfer F (2008) J Phys Condes Matter 20:11

39. Somorjai GA (1994) Annu Rev Phys Chem 45:721

40. Tian HC, Liu L, Wen YH (2009) Acta Phys Sin 58:4080

41. Hopstaken MJP (2000) In: Elementary reaction kinetics and lateral interactions in the catalytic reaction between $\mathrm{NO}$ and $\mathrm{CO}$ on rhodium surfaces. Schuit Institute of Catalysis, Eindhoven University of Technology, Eindhoven, The Netherlands

42. Kim YJ, Thevuthasan S, Herman GS, Peden CHF, Chambers SA, Belton DN, Permana H (1996) Surf Sci 359:269

43. Loffreda D, Simon D, Sautet P (1998) J Chem Phys 108:6447

44. Oh SH, Fisher GB, Carpenter JE, Goodman DW (1986) J Catal 100:360

45. Zaera F, Gopinath CS (2000) Chem Phys Lett 332:209

46. Hopstaken MJP, Niemantsverdriet JW (2000) In: The 46th international symposium of the American Vacuum Society, Seattle, Washington, USA 